Revue des patrimoines

46 | 2022

Le patrimoine de la Justice

\title{
Le bagne portuaire de Rochefort (1766-1852) : un patrimoine disparu
}

The harbour prison of Rochefort (1766-1852), a lost heritage

\section{Philippe Duprat}

\section{(2) OpenEdition}

\section{Journals}

Édition électronique

URL : https://journals.openedition.org/insitu/33483

DOI : $10.4000 /$ insitu.33483

ISSN : 1630-7305

Éditeur

Ministère de la Culture

\section{Référence électronique}

Philippe Duprat, « Le bagne portuaire de Rochefort (1766-1852) : un patrimoine disparu », In Situ [En ligne], 46 | 2022, mis en ligne le 18 janvier 2022, consulté le 03 février 2022. URL : http:// journals.openedition.org/insitu/33483; DOI : https://doi.org/10.4000/insitu.33483

Ce document a été généré automatiquement le 3 février 2022



In Situ Revues des patrimoines est mis à disposition selon les termes de la licence Creative Commons Attribution - Pas d'Utilisation Commerciale - Pas de Modification 4.0 International. 


\title{
Le bagne portuaire de Rochefort (1766-1852) : un patrimoine disparu
}

\author{
The harbour prison of Rochefort (1766-1852), a lost heritage
}

\section{Philippe Duprat}

1 Le bagne de Rochefort (Charente-Maritime), qui a fonctionné de 1766 à 1852 au cœur du système productif de l'arsenal, a disparu de la mémoire rochefortaise malgré ses quatre-vingt-deux ans d'existence et les quelque 25000 forçats qu'il a hébergés, dont plus de la moitié sont morts en détention: aucun vestige du monument ne subsiste ${ }^{1}$, l'oubli est quasi total dans la mémoire collective et l'on chercherait en vain la moindre plaque commémorative. Comment s'explique ce phénomène?

2 Les archives sont pourtant abondantes, prioritairement au Service historique de la Défense (SHD) à Vincennes ${ }^{2}$ et à l'antenne du SHD de Rochefort ${ }^{3}$. D'utiles compléments existent aux archives départementales de la Charente-Maritime, à la médiathèque de La Rochelle, et dans les services d'archives de Rochefort (Archives municipales ${ }^{4}$, médiathèque, musée Hèbre), sans oublier le WEBSIG géohistorique-Rochefort ${ }^{5}$. Ce sont là les sources principales du présent article.

3 Quant aux études sur le bagne de Rochefort, elles demeurent rares et très incomplètes, malgré une littérature pléthorique sur les bagnes en général, qu'il convient d'exploiter avec prudence, nous le verrons. En l'absence de vestiges visibles, seule l'archéologie pourrait apporter d'autres ouvertures mais la question demeure délicate dans une « ville nouvelle » où la recherche dans ce domaine est un phénomène très récent ${ }^{6}$. Dans l'immédiat, c'est donc l'histoire qui permet de lever le voile sur cette institution à la fois terrible et fascinante, que l'on s'est empressé d'effacer de la mémoire dès 1852 .

4 C'est en juin 1763 que se fait sentir «la nécessité d'avoir des forçats au port de Rochefort, pour y servir comme journaliers, ainsy que cela se pratique à Brest et à Toulon ${ }^{7}$ ». La date n'est pas anodine : elle correspond à la fin de la guerre de Sept Ans, 
et à la perte presque complète pour la France de son premier empire colonial en Amérique du Nord (Nouvelle-France et est de la Louisiane), au bénéfice de l'Angleterre.

5 La France, qui sort très affaiblie de ce conflit et extrêmement endettée, lance une politique de reconstitution de sa marine décimée. À Rochefort, les années de guerre (1756-1763) ont été difficiles, malgré la mise en chantier d'une vingtaine de navires dès 1756. Mais l'arsenal subit de plein fouet les succès navals britanniques de 1758-1759 en Amérique du Nord ${ }^{8}$. C'est dans ce contexte de perte d'influence mondiale et d'affaiblissement économique, militaire et maritime de la France que l'on envisage la création d'un bagne dans l'arsenal de Rochefort, à l'image de Toulon et de Brest, afin de bénéficier de la main-d'œuvre gratuite des forçats, pour amorcer à moindres frais le redressement.

\section{Le bagne du XVIII ${ }^{\mathrm{e}}$ siècle}

\section{Les atermoiements des origines : bagne flottant / bagne terrestre}

Dès 1763, à Rochefort, on envisage de loger les forçats sur deux galères qui permettraient également de remorquer sur la Charente les vaisseaux de ligne en cours d'armement. L'idée paraît séduisante mais la configuration de la Charente et les essais faits à Toulon en juillet 1763 ne sont pas concluants. Provisoirement abandonnée, l'idée d'un bagne n'est relancée qu'en mai 1766. La volonté du roi est « de faire établir à Rochefort une chiourme composée de 5 à 600 forçats pour servir, comme à Brest et à Toulon, aux travaux du port et suppléer de plus aux gens de liberté qu'on y employe, souvent mesme par corvée, à la cordelle pour conduire en rivière les vaisseaux qui sont en armement ${ }^{9} »$. Dès l'origine, le bagne de Rochefort se distingue donc de ceux de Brest et Toulon par sa spécificité : la double nature des travaux de fatigue pour les forçats, ceux - classiques - de l'arsenal, mais aussi le halage des navires tout au long des $21 \mathrm{~km}$ qui séparent Rochefort de l'embouchure de la Charente ${ }^{10}$.

7 Pour constituer la chiourme, on prévoit d'héberger 500 à 600 forçats sur deux prames (bateaux de transport à fond plat) mouillant dans la partie sud de l'arsenal, où sont disposées presque toutes les structures de production (cales de construction des navires, forges, ateliers, magasins généraux et particuliers, fosses aux mâts...), zone très marécageuse, bien circonscrite entre la ville et la Charente, et ceinturée par un réseau de murs et de canaux, qui sont autant de gages de sécurité [fig. 1]. 
Figure 1



Emplacement du futur bagne. Plan de la Ville et du Port de Rochefort pour servir aux projets de 1754 - 28 Aoust 7753 (extrait), conservé au service historique de la Défense, château de Vincennes (VI DD2, 708 II 21b).

Reproduction SHD Vincennes.

8 Finalement, sur l'avis motivé de Jean-François Testanières, commissaire des chiourmes à Brest ${ }^{11}$, on renonce au bagne flottant. Le ministre approuve « [le parti] d'établir des forçats dans le hangar des futailles ", solution provisoire avant l'installation définitive du bagne dans « le bastiment de la vieille poudrière, dite Saint-Maurice ». En réalité, le « hangar des futailles » marque l'implantation définitive du bagne de Rochefort, au plus près de l'appareil productif.

9 À la différence de Brest ${ }^{12}$, il n'y a donc pas à Rochefort d'architecture typique du bagne portuaire mais un aménagement improvisé et guidé par un impératif financier de réduction des coûts.

\section{Le premier bagne}

10 Le « hangar des futailles », également appelé « hangar aux bois », créé en 1759 derrière les Magasins généraux, au-delà d'un canal central qui existe depuis 1687, doit être rapidement aménagé car le temps presse, l’arrivée des premiers forçats étant prévue pour septembre 1766 : il faut élargir la structure, paver le sol et installer les «taulas ${ }^{13}$ » pour le couchage d'environ 500 forçats.

Le bâtiment est prêt en octobre 1766 pour accueillir la chaîne ${ }^{14}$ de Guyenne, arrivée le 11 octobre (128 hommes), puis la chaîne de Brest, arrivée le 21 (400 hommes), soit un effectif total de 528 hommes $^{15}$. Tout se fait un peu dans l'urgence: les effectifs de surveillance ne sont pas encore au complet, les vêtements et chaussures pas encore livrés, et au 18 octobre, les bureaux et magasins à adjoindre au bâtiment de la chiourme pas encore construits. 
12 Un plan détaillé, daté de 1766, montre la structure originelle du bagne [fig. 2] : un édifice rectangulaire s'ouvrant sur une cour fermée par deux ailes latérales et un large canal doté d'un pont. Le mur de façade est en bois. L'édifice principal, divisé en deux parties et doté de cellules à chaque extrémité, est réservé à l'hébergement des forçats avec ses «taulas ». Il est appelé « salle Saint-Gilles ». Les ailes latérales concernent les services de la garde, des magasins et des bureaux.

Figure 2

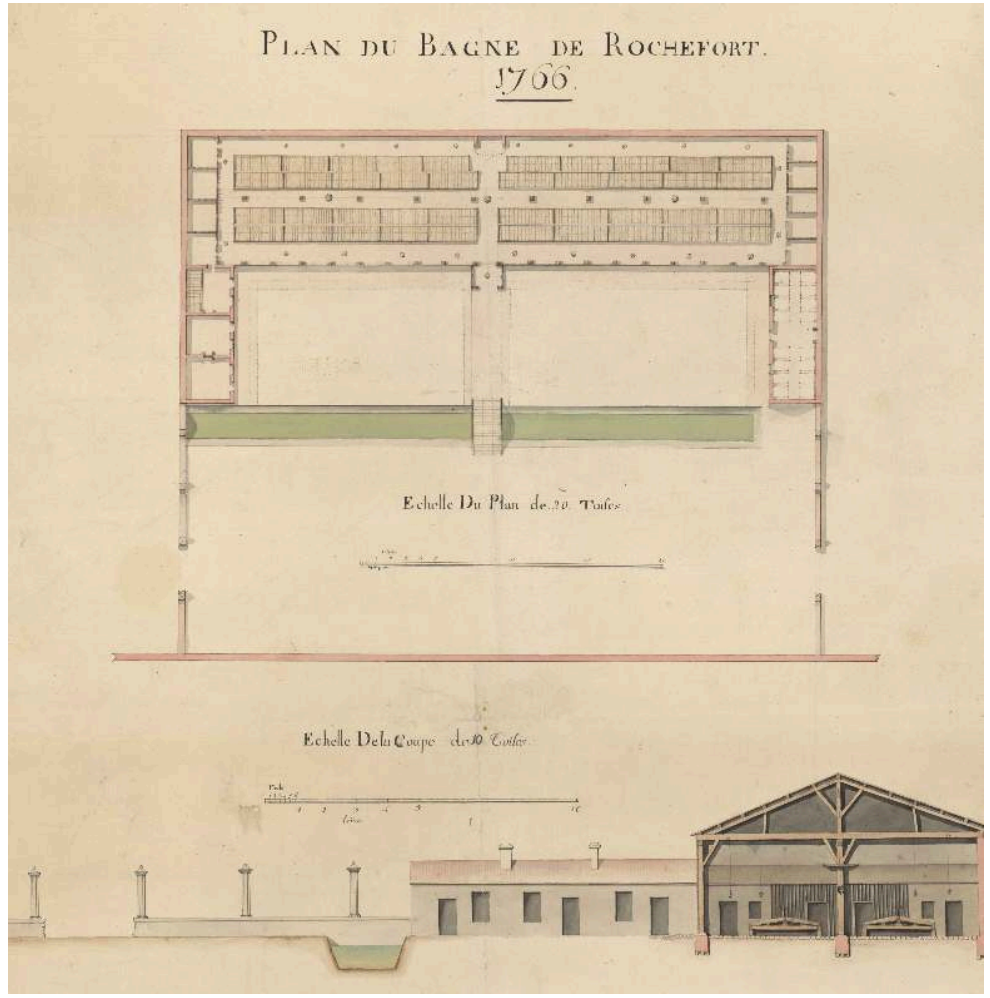

Plan du bagne de Rochefort - 1766 conservé au service historique de la Défense, château de Vincennes (VI DD2, 695 48).

Reproduction SHD Vincennes.

Dès 1767, un nouveau bâtiment apparaît au nord dans le prolongement axial de la salle Saint-Gilles ; c'est le « hangar de l'artillerie » qui permettra une extension du bagne : la structure définitive de l'établissement pénitentiaire est donc en place [fig. 3]. Sur le plan de 1769, le grand canal central, qui délimitait le bagne du côté de la Charente, est déjà comblé, libérant une vaste cour devant la salle Saint-Gilles : il est détourné à l'arrière des bâtiments. 
Figure 3

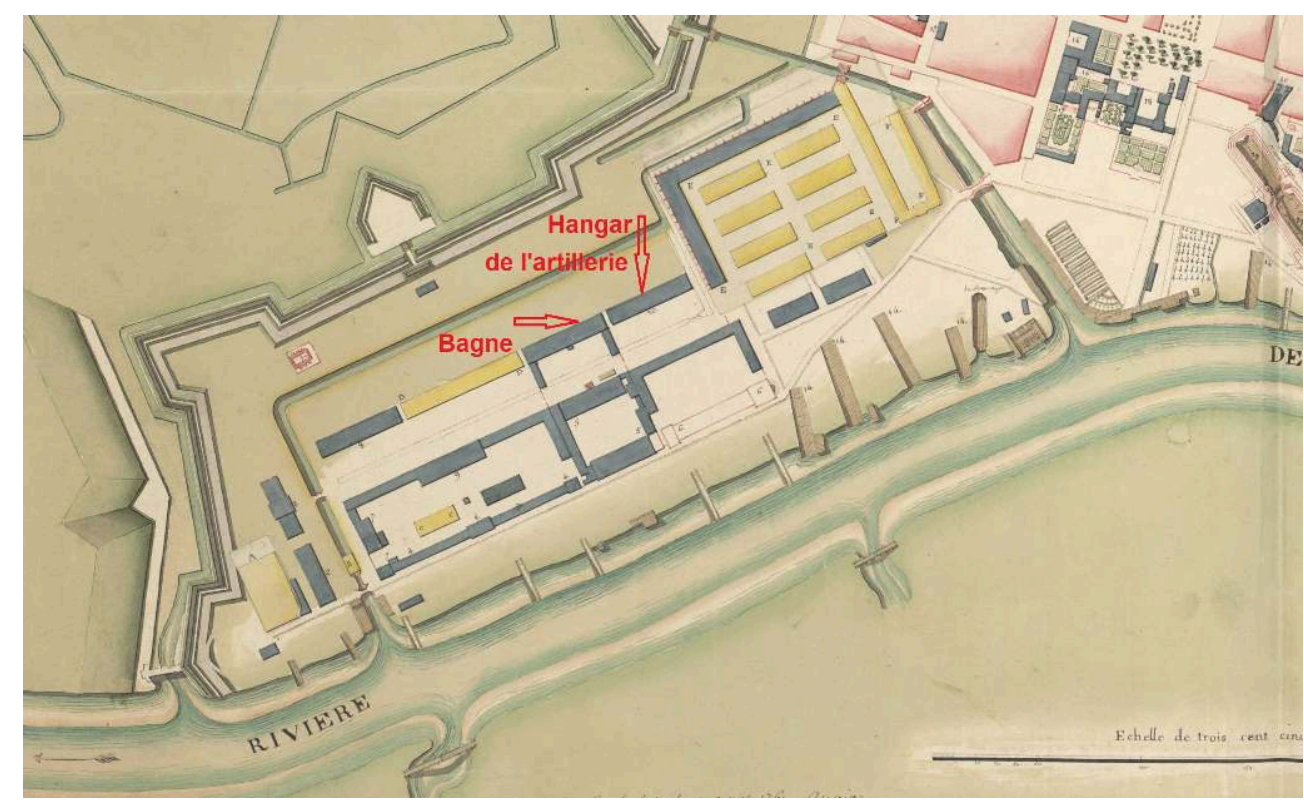

Plan de la ville et du port de Rochefort - 19 avril 1769 (extrait) conservé au service historique de la Défense, château de Vincennes (fonds Nivard, VI SHDMD0071783 P).

Reproduction SHD Vincennes.

À partir de 1774 sont créés, dans cette partie arrière, les jardins du bagne, destinés à compléter la nourriture en légumes des forçats pour combattre le scorbut. Le canal détourné ceinture et assainit toute cette zone humide [fig. 4]. Un petit hôpital provisoire, signalé par le commissaire Jacques-Christophe Millet ${ }^{16}$ près du pont de la Cloche, est visible sur le plan de 1774 , sous la dénomination d'« hôpital des forçats convalescents ».

Le bagne rochefortais conserve cette configuration jusqu'à la guerre d'Indépendance américaine, avec un effectif qui varie entre 550 et 700 forçats (capacité maximale des locaux, selon l'intendant): de 1766 à 1775, l'activité de l'arsenal reste modeste (seulement 13 navires construits). 
Figure 4

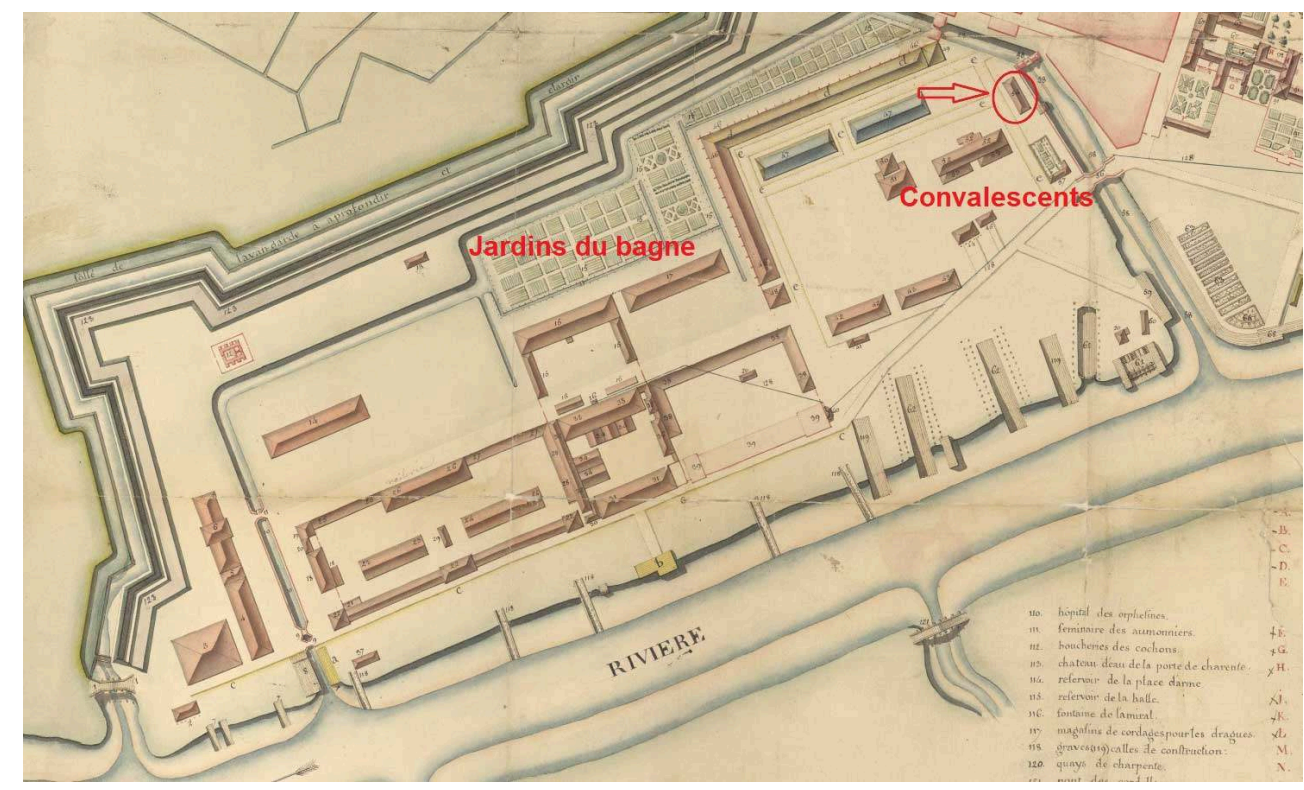

Plan de la Ville et du Port de Rochefort relatif aux projets de 1775 - 20 décembre 1774 (extrait) conservé au service historique de la Défense, château de Vincennes (DD2, VI SHDMD07002527 P).

Reproduction SHD Vincennes.

\section{La fin de l'Ancien Régime}

Avec la guerre d'Indépendance américaine (1776-1783), l'arsenal de Rochefort retrouve des couleurs après une longue période de léthargie. Ce sont 50 navires ${ }^{17}$ qui sortent des cales dans cette courte période, dont 7 mis en chantier dès 1776. Comme à Brest et à Toulon, les besoins de main-d'œuvre sont énormes ${ }^{18}$ : les effectifs de la chiourme grimpent à 1060 hommes en 1776 pour atteindre 1250 en $1781^{19}$. Pour accueillir l'arrivée massive de la main-d'œuvre des forçats, il faut doubler la capacité d'accueil. Le « hangar de l'artillerie », qui jouxte la salle Saint-Gilles, lui est immédiatement accolé et prend le nom de «salle Saint-Antoine » en 1777, chacune des deux salles pouvant contenir 550 hommes.

Le petit hôpital du pont de la Cloche (qui servait en réalité de salle des convalescents), devenu trop exigu, est supprimé en 1780, et transféré au " Coquerond », ancienne salle aux artifices, située au sud-ouest près du mur d'enceinte. La nouvelle salle des convalescents est agrandie et appelée «salle Saint-Jacques » [fig. 5]. Elle peut contenir jusqu'à 240 convalescents et fonctionnera jusqu'à la fin du bagne. 
Figure 5

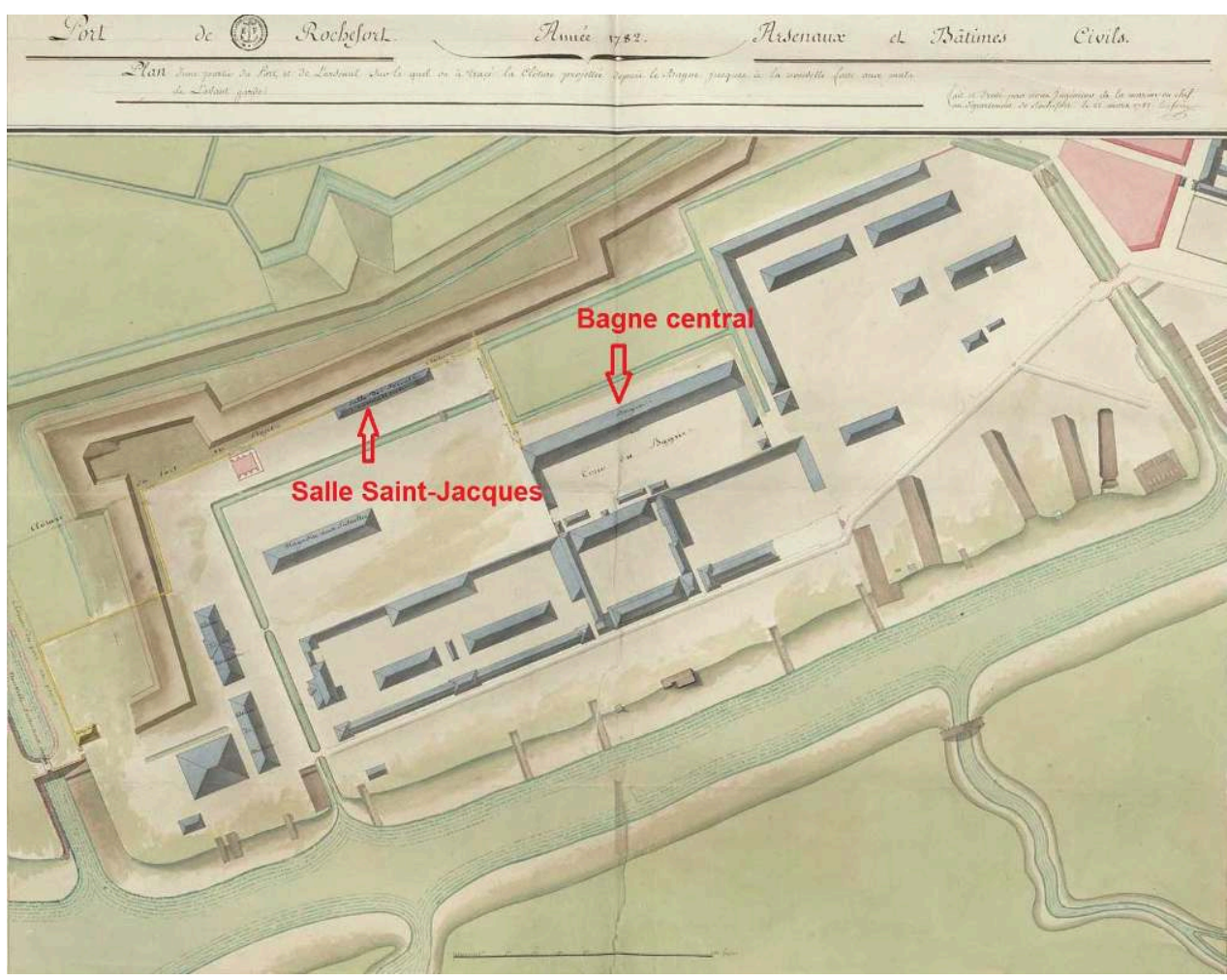

Port de Rochefort - Année 1782 - Arsenaux et Bâtimens Civils - 22 mars 1782 (extrait), plan conservé au service historique de la Défense, château de Vincennes (fonds Nivard, VI SHDMD07001702 P).

Reproduction SHD Vincennes.

L'aménagement définitif du corps central du bagne, doté des deux grandes salles contiguës flanquées de deux ailes, est réalisé entre 1780 et 1782 : la configuration d'ensemble ne variera plus jusqu'en 1852, avec une façade en pierre d'environ 150 mètres de long.

\section{Le bagne du $\mathrm{XIX}$ siècle}

\section{Durant la Révolution et l'Empire}

Les guerres de la Révolution et du Consulat relancent à nouveau les activités de l'arsenal, avec la mise en chantier de 31 navires dont 14 grands vaisseaux et 9 frégates. Sous l'Empire, entre 1804 et 1814, 65 navires sont construits à Rochefort.

Malgré la pénurie d'ouvriers libres, les effectifs du bagne restent stables jusqu'en 1796, puis repartent à la hausse ${ }^{20}$, mais avec le gouvernement thermidorien s'amorce une solution inédite reprise et amplifiée par les régimes successifs : la création de nouveaux bagnes (Lorient en 1794, Le Havre en 1798, Cherbourg en 1802), notamment dans les ports des territoires vassaux de l'Empire (Nice, Villefranche, Civitavecchia dans le Latium, La Spezia en Ligurie, Anvers...). Les effectifs des bagnes français passent de 4000 condamnés en 1795 à 16000 en 1814 : on a pu parler de "petit goulag ${ }^{21}$ » à l'échelle européenne du Grand Empire. On y trouve pêle-mêle condamnés de droit commun, contrebandiers, soldats, insoumis et patriotes allemands ou espagnols ${ }^{22}$, etc. 
Comme après la guerre d'Amérique, le retour à la paix s'accompagne d'une chute globale du nombre des forçats (9 000).

21 À Rochefort, comme à l'habitude, on s'adapte avec les locaux disponibles, la capacité d'accueil n'étant pas extensible : ces bâtiments sont le plus souvent utilisés à la limite de leur capacité, l'objectif étant de minimiser le coût journalier du forçat en maximisant le taux d'occupation. Le seuil de capacité (c'est-à-dire les places de couchage sur le taulas), fixé à 1500 hommes, est régulièrement dépassé. Devant le surplus de forçats qu'il faut loger dans les deux salles centrales, le commissaire Millet imagine de " gêner un peu les forçats » en réduisant légèrement, de 18 à 17 pouces (soit de 49 à $46 \mathrm{~cm}$ environ), l'espace individuel de couchage, optimisation qui lui permet de gagner 152 places!

Il faut aussi pouvoir héberger le nombre de convalescents qui s'accroît proportionnellement ${ }^{23}$ et que la salle Saint-Jacques, bien qu'agrandie, ne peut plus accueillir : en 1801, on ouvre à cet effet la «salle neuve » (un magasin aux bois de construction), qui sera continuellement occupée jusqu'en 1833 [fig. 6].

Figure 6

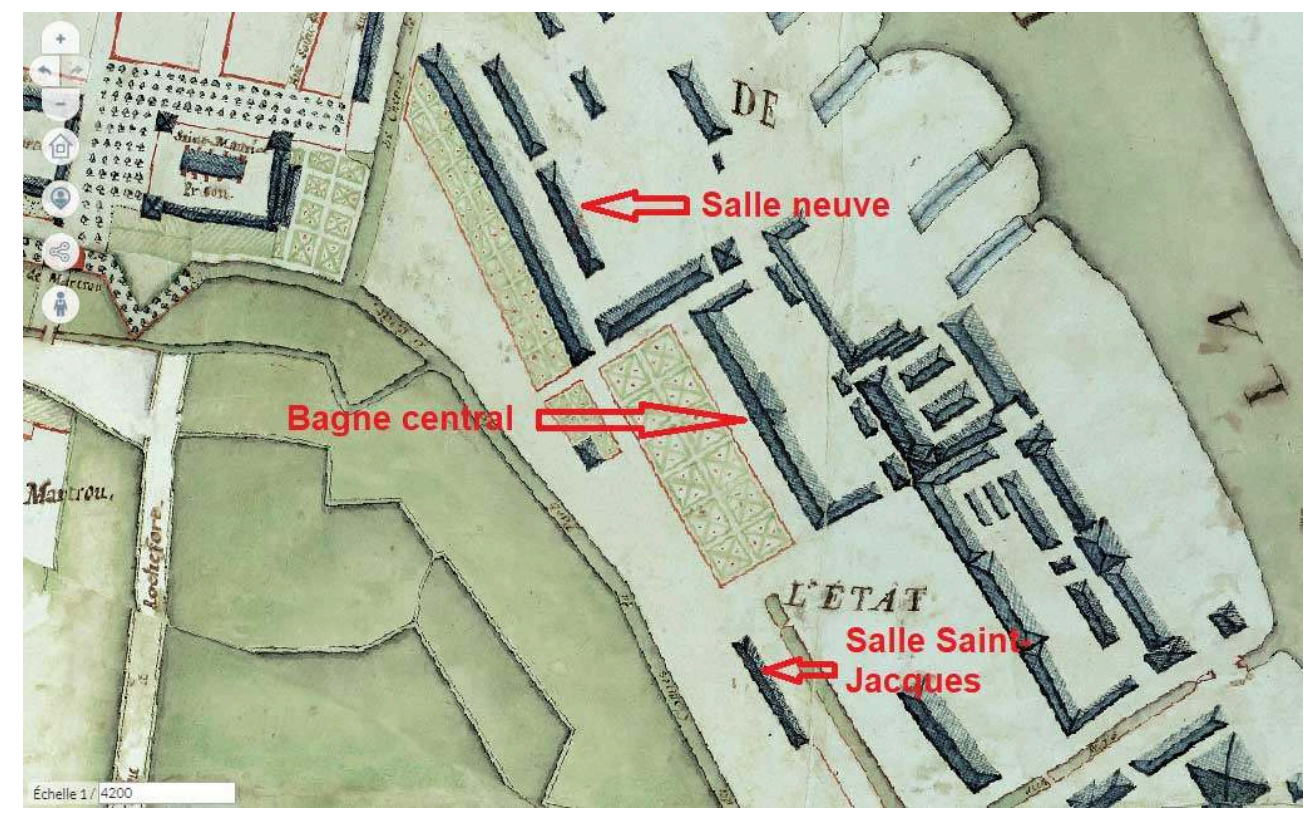

Plan de Rochefort levé en 1871 (extrait), conservé aux archives départementales de la CharenteMaritime (AD 17, 5 Fi Rochefort 6).

DAO sur le Websig géohistorique sur Rochefort : https://geoapps.huma-num.fr/adws/app/ 0101e6d0-628e-11e8-8be3-df0fcbe237ee/ [lien valide novembre 2021].

\section{Le bagne de la Restauration}

Les lieux principaux du bagne, désormais fixés, évoluent peu durant cette période. Pourtant les effectifs des forçats augmentent, après la baisse liée au retour de la paix et à la quasi-interruption des constructions navales entre 1814 et 1820 : les chiffres oscillent entre 1300 et 1800 bagnards, avec un pic à 2057 en 1824, constituant le record d'occupation des lieux. Cette explosion quantitative s'explique par la sévérité accrue des jurés d'assises envers les voleurs (qui constituent plus de $80 \%$ des bagnards, toutes périodes confondues ${ }^{24}$ ) durant cette période marquée par le conservatisme 
politique réactionnaire. C'est aussi une période de transition technologique, la voile se taillant encore la part du lion : la propulsion à vapeur demeure balbutiante ( 5 navires sur un total de 31). La main-d'œuvre issue du bagne reste donc indispensable aux travaux de l'arsenal.

En l'absence de tout schéma directeur pour le bagne à Rochefort, c'est toujours le principe de l'adaptation ponctuelle aux besoins qui prédomine, et l'obsession du moindre coût. On s'installe dans un provisoire plus ou moins improvisé. En 1821, face à l'afflux de nouveaux condamnés, l'ingénieur Mathieu propose de surélever d'un étage le bâtiment central du bagne, abritant les deux salles principales, projet sans suite. Mais il faut bien loger les forçats : à partir de 1822 et jusqu'en 1829, un second bagne occupe une aile de la caserne Martrou.

Durant cette période, l'établissement pénitentiaire atteint son extension géographique maximale, en se répartissant sur cinq bâtiments (y compris l'hôpital de la Marine, où l'on soigne les forçats malades ou blessés) : un noyau central $\left(n^{\circ} 1\right)$ et quatre satellites, dont deux à l'extérieur de l'enceinte de l'arsenal. Deux bâtiments sont réservés à $l^{\prime}$ hébergement ( ${ }^{\circ} 1$ : salles Saint-Gilles et Saint-Antoine ; $n^{\circ} 5$ : caserne Martrou), deux autres aux soins médicaux $\left(\mathrm{n}^{\circ} 2\right.$ : hôpital de la Marine; $\mathrm{n}^{\circ} 3$ : salle Saint-Jacques). La salle neuve $\left(n^{\circ} 4\right)$ sert de variable d'ajustement (convalescence ou hébergement). Le plan d'ensemble souligne l'éclatement de la structure [fig. 7].

Figure 7

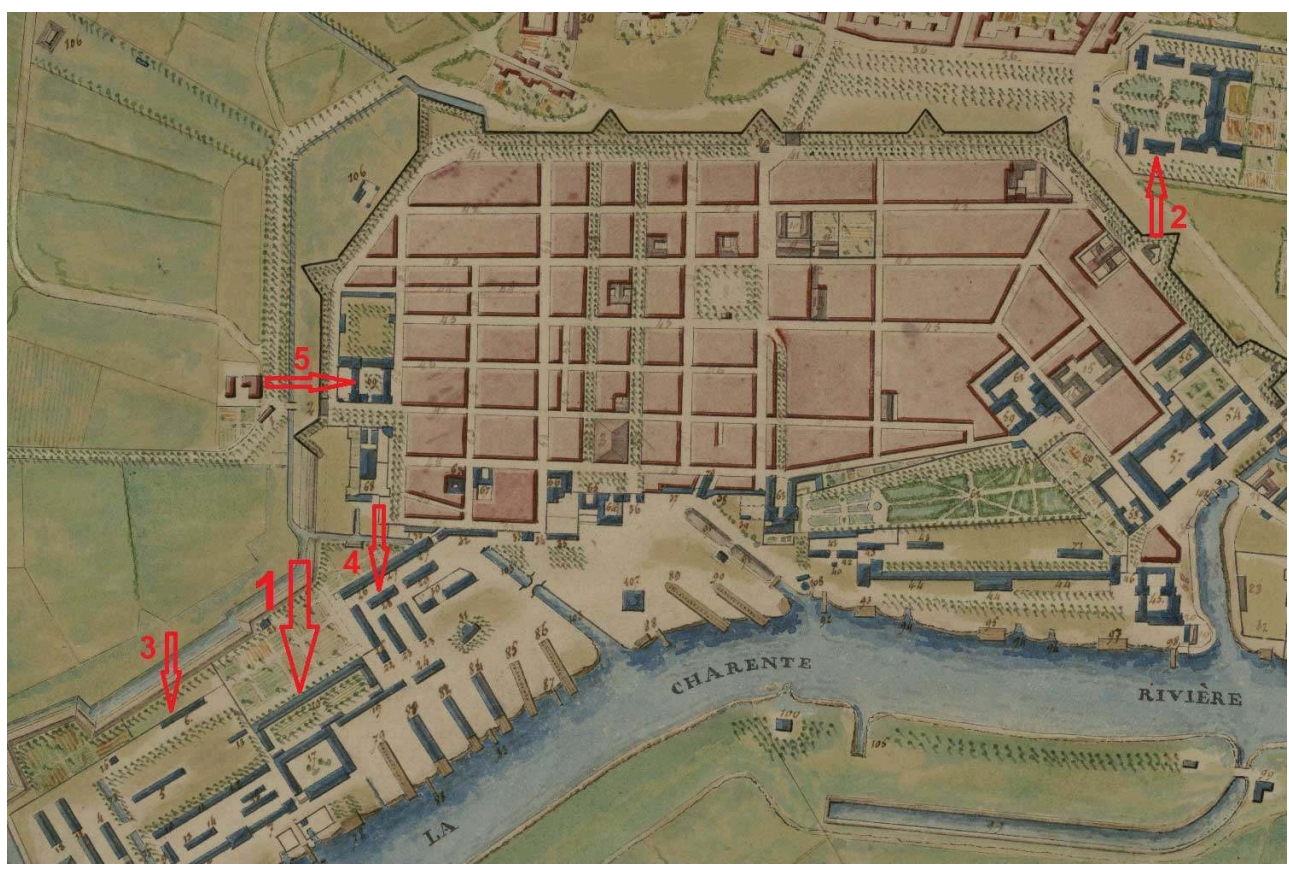

L'extension maximale du bagne de Rochefort entre 1821 et 1833. Plan de la ville et port de Rochefort (extrait), 1830, conservé à la médiathèque d'agglomération La Rochelle (3 pl 37).

Reproduction médiathèque d'agglomération La Rochelle.

\section{Sous la Monarchie de Juillet}

Après 1830, les effectifs du bagne baissent, se maintenant jusqu'à la fin à un étiage relativement bas (de 900 à 1141 hommes). Ce reflux du nombre des forçats peut être 
mis en relation avec la révolution de la vapeur, qui supplante définitivement la voile, même si le plan-relief de Touboulic (réalisé vers 1834) montre encore une large prédominance des navires à voile [fig. 8].

Figure 8

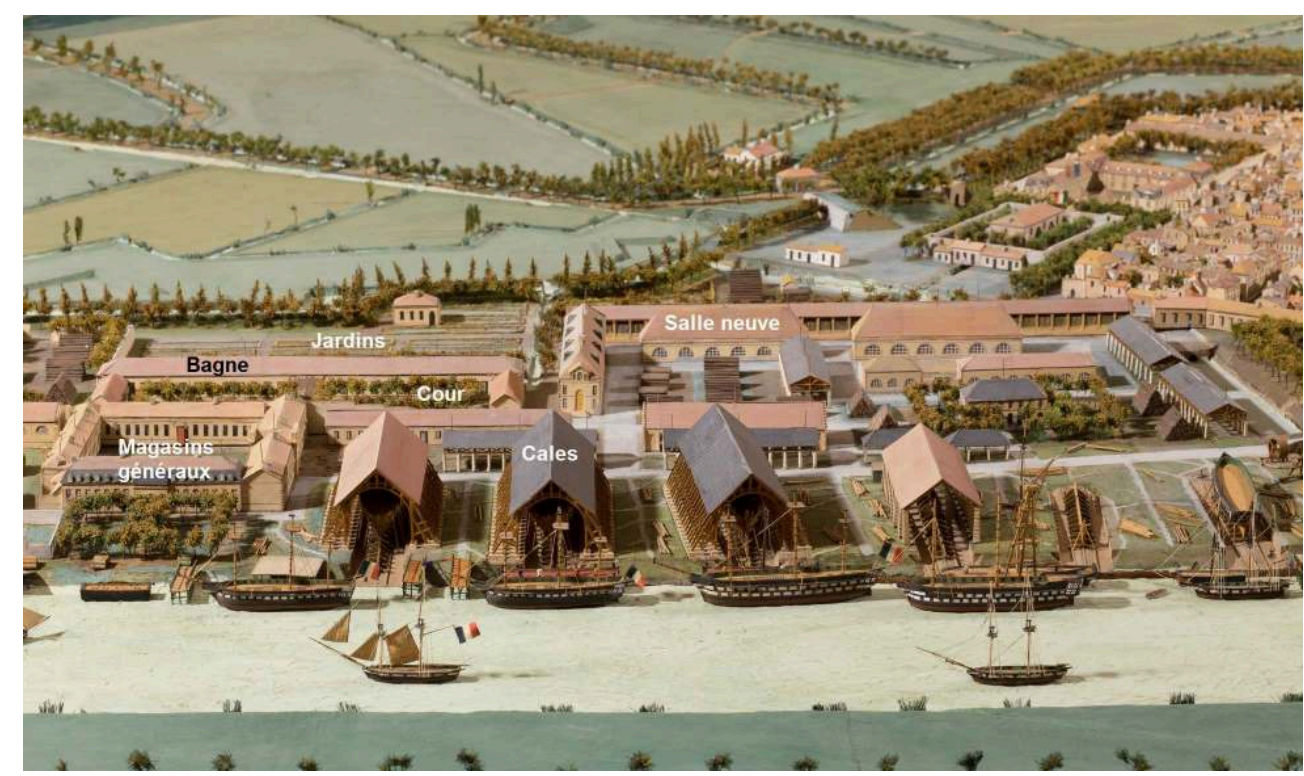

Plan relief de Rochefort, le bagne, P.-M. Touboulic, 1834, conservé au Musée Hèbre de Rochefort ( $\mathrm{N}^{\circ}$ inv. 2007.8.43)

Reproduction Musées-Municipaux Rochefort 17.

Sur les 32 navires de guerre construits dans cette période, 26 fonctionnent à la vapeur (à roues, à hélices ou mixtes). Parmi les bateaux utilitaires, on note la construction de deux remorqueurs (à vapeur) entre 1839 et 1843 : la disparition de la cordelle est actée. La main-d'œuvre de l'arsenal se spécialise peu à peu dans la vapeur et le fer, marginalisant celle des condamnés, essentiellement vouée aux travaux de force en liaison avec le bois et le dévasement manuel des formes et fosses aux mâts.

À Rochefort, le bagne se replie donc dans ses murs originels (hormis l'hôpital de la Marine où sont toujours soignés les forçats), se limitant de plus en plus à une structure d'enfermement. Après l'intermède de la caserne de Martrou, la salle neuve est à son tour rendue aux ateliers de l'arsenal en 1833 ; désormais, tous les bagnards logent dans les deux salles du bâtiment central [fig.9]. Seuls les convalescents, invalides et infirmiers continuent d'être hébergés dans la salle Saint-Jacques. 




Plan du bagne de Rochefort, attribué à Clemens vers 1845, conservé au Musée Hèbre de Rochefort ( $N^{\circ}$ inv. 001.2.1).

Reproduction Musées-Municipaux Rochefort 17.

\section{L'évolution de l'espace pénitentiaire}




\section{La fin du bagne de Rochefort} l'Empire.

Le développement des nouvelles technologies de la vapeur et du fer se poursuit inexorablement et s'accélère au début des années 1850 , rendant obsolète la maind'œuvre peu qualifiée des forçats, de plus en plus inadaptée au fonctionnement moderne d'un arsenal. La fin du bagne continental est programmée.

Aux arguments économiques (coût et manque de rentabilité) s'ajoutent les arguments sécuritaires (la porosité de l'enceinte), ainsi que, dès 1826-1827, les campagnes d'opinion dénonçant la nocivité des bagnes, demandant leur "transportation » outremer. Les projets de déportation réapparaissent après les journées de 1848 , avec la peur que suscitent les classes laborieuses («dangereuses»). Le 30 mai 1850, le Princeprésident souligne le poids de l'entretien de 6000 forçats dans le budget de la nation, la menace qu'ils font peser sur la société, l'opportunité d'utiliser les progrès de la colonisation française. La Marine, qui a aussi les colonies sous sa tutelle, ne s'oppose pas à ces vues. En octobre 1851, le Prince-président est accueilli chaleureusement à Rochefort, le conseil municipal votant à cette occasion un vœu de rétablissement de

Le bagne de Rochefort est supprimé dès 1852. Brest ferme en 1858 et Toulon, définitivement, en 1873. Le ministre de la Marine organise les modalités de la fermeture entre février et juin 1852 : remplacement des bagnards-ouvriers par des journaliers, transfert de la chiourme par bateaux à vapeur (244 forçats pour Brest; 514 à destination de Cayenne).

Cette fermeture ne semble pas avoir soulevé d'émotion à Rochefort. Le sujet n'a même pas été évoqué au conseil municipal. Le journal local Les Tablettes des deux Charentes donne l'information en quelques lignes, parmi d'autres, comme l'arrivée à Rochefort d'un bateau à vapeur. Il précise surtout les avantages proposés aux bagnards volontaires pour le départ en Guyane: "Toutes les dispositions de ce décret sont dictées par un sentiment de juste sévérité et d'humanité ${ }^{30}$. " Le remplacement des bagnards par des ouvriers-journaliers ne peut apparaître que comme positif même si «cette mesure [la fermeture du bagne] enlève à l'industrie locale une source de revenus certains, elle sera cependant, au point de vue moral, approuvée sans réserve par tous les esprits droits et éclairés ». C'est donc le " point de vue moral » qui prévaut, avant toute considération de nature économique, aussi bien pour les patrons et commerçants que pour les ouvriers.

7 Un plan précis est dressé en 1852 pour servir d'état des lieux, permettant d'envisager une reconversion rapide [fig. 10]. 
Figure 10



Plan du bagne à sa fermeture en 1852. Plan de l'état actuel des bâtiments occupés par l'ancien bagne (1852) conservé au service historique de la Défense, Rochefort (MR K 1081 0001).

Reproduction SHD Rochefort.

\section{Reconversion industrielle, histoire et mythe}

\section{Les locaux du bagne réaffectés à l'arsenal}

Après le départ des forçats, les locaux du bagne sont réintégrés aux services techniques de l'arsenal. Le grand bâtiment central (qui abritait la salle Saint-Gilles et la salle SaintAntoine), flanqué de ses deux ailes, est transformé en chaudronnerie (salle SaintAntoine) et en magasins (salle Saint-Gilles), tandis que la grande cour est dotée de petits bâtiments et ateliers divers (hangars, dépôt des pompes, machine à scier le bois, etc.). Des magasins et dépôts occupent l'ancienne salle Saint-Jacques [fig. 11]. 
Figure 11



Les bâtiments de l'ancien bagne en 1885. Atlas des ports de France - Port de Rochefort - 1885 (extrait), conservé aux archives Société de Géographie de Rochefort.

Reproduction Société de Géographie de Rochefort.

Une photo datée de 1882 [fig. 12], conservée au SHD de Rochefort, montre l'ancienne forge du bagne (qui figure sur le plan de 1852) transformée en atelier de petite chaudronnerie. 
Figure 12

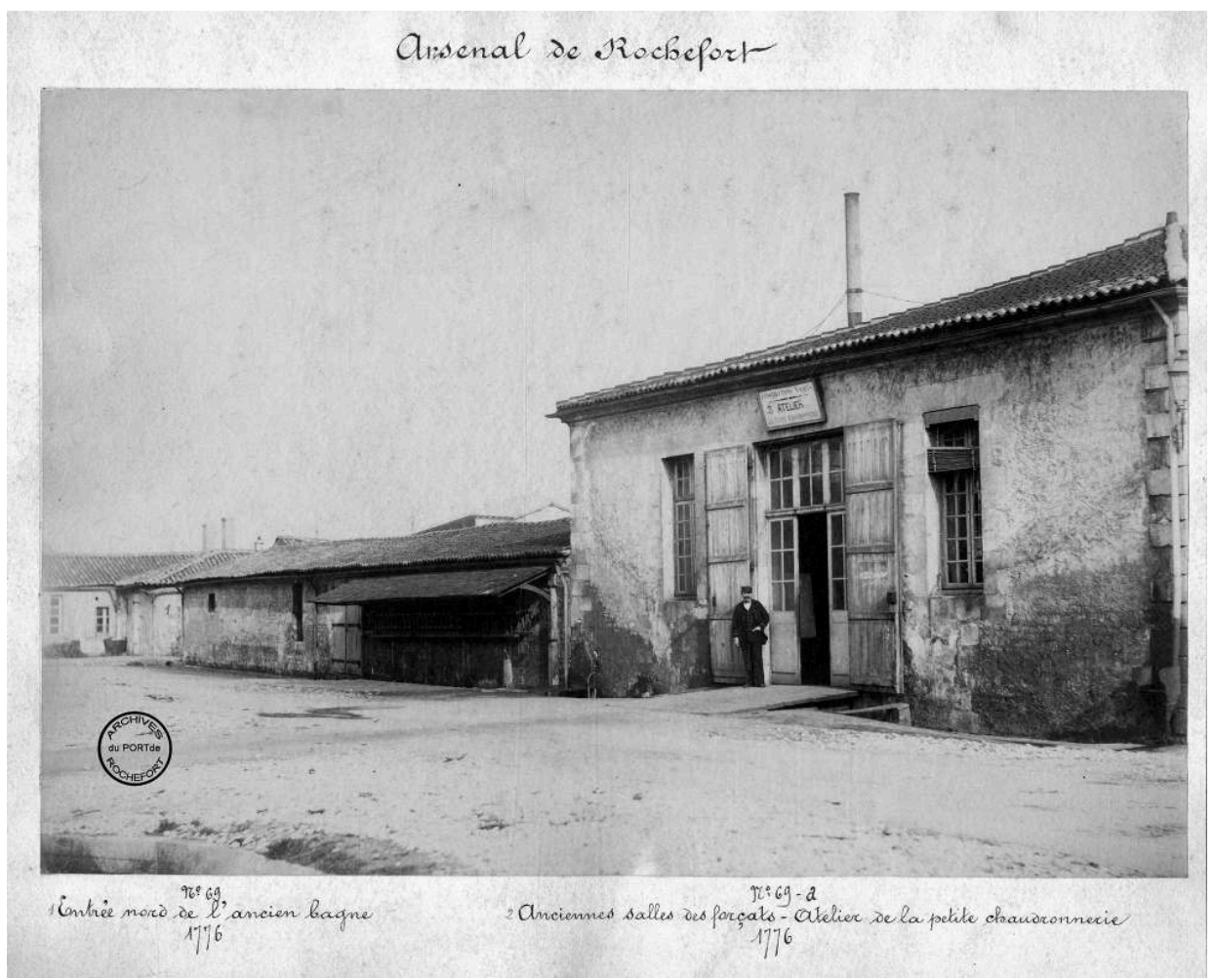

L'ancienne forge du bagne, transformée en atelier de petite chaudronnerie. Arsenal de Rochefort entrée nord de l'ancien bagne (1882), conservé au service historique de la Défense, Rochefort (3 KW).

Reproduction SHD Rochefort.

L'arsenal ferme définitivement en 1927 mais la plupart des bâtiments importants sont peu à peu remis par la Marine aux Domaines et réaffectés à de nouvelles activités. La réparation navale est reprise par la société Goldenberg en 1928 (qui dépose son bilan en 1930), puis par la Société industrielle rochefortaise (SIR), qui ferme ses portes en 1936. Zodiac prend la succession en 1937 et commence à fabriquer des dirigeables et ballons sur la zone sud de l'arsenal à partir de 1939 (commandes militaires), renforçant l'implantation de l'aéronautique à Rochefort. Celle-ci avait commencé dès juillet 1935, avec l'installation de l'usine d'aviation Lioré et Olivier (montage d'hydravions), nationalisée au début de 1937 (SNCASO ${ }^{31}$ ). Les bâtiments de l'ancien bagne servent d'entrepôts.

\section{L'occupation allemande et les premières destructions}

En 1940, nombre de bâtiments de l'arsenal (dont l'ancien bagne) sont réquisitionnés par les Allemands car Rochefort est en zone interdite ${ }^{32}$. Zodiac réduit fortement son activité, fabricant des parachutes destinés aux forces d'Occupation. En août 1944, peu avant leur départ, les Allemands détruisent les infrastructures du port qu'ils minent ou incendient : c'est notamment le sort de toutes les écluses des formes, de la corderie, d'une partie des magasins et ateliers et de l'ancien bagne. Une photo aérienne datée de 1947 en montre clairement les ruines [fig. 13]. 


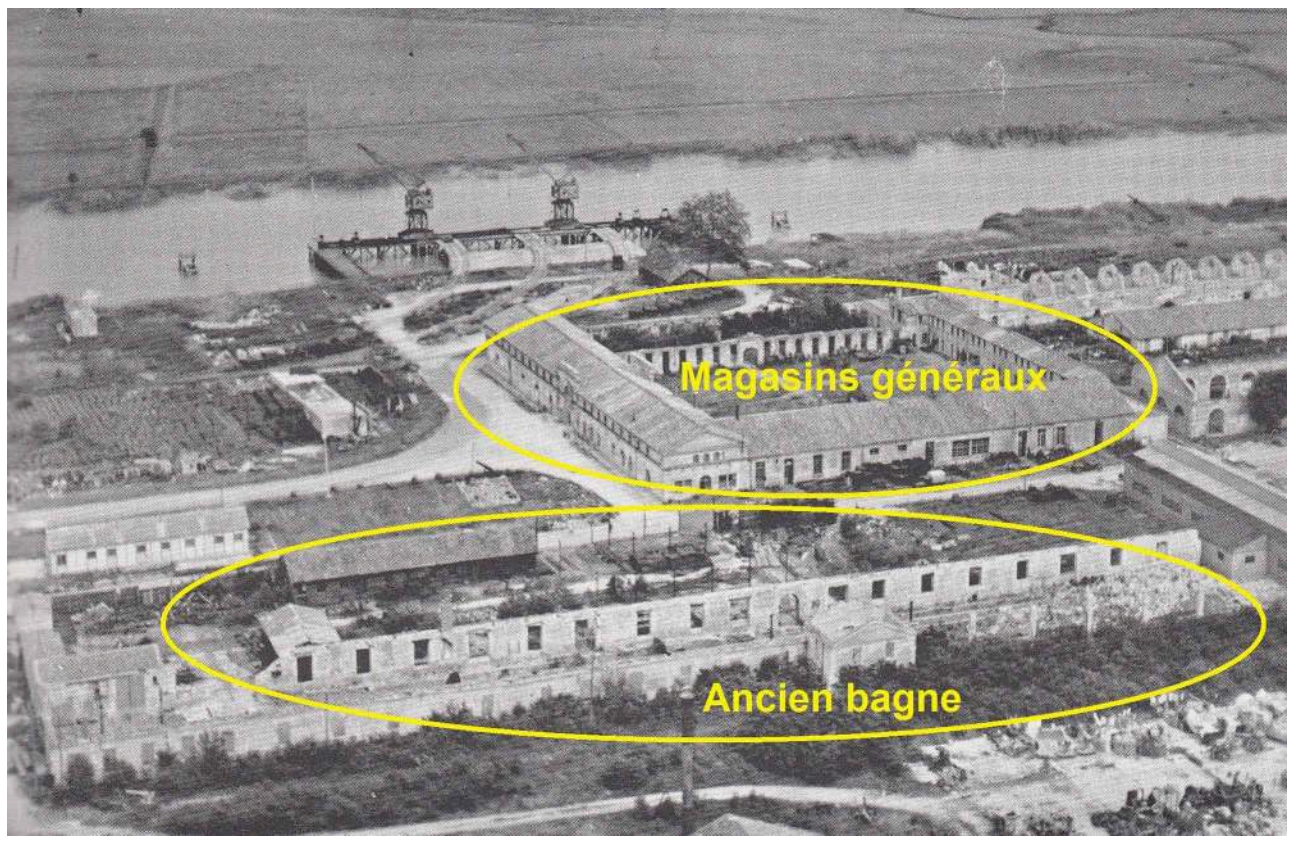

Vue aérienne de l'arsenal de Rochefort, partie sud (1947), extraite de Rochefort, trois siècles en images, CALC Rochefort, 1981, p. 157

(c) Photographe inconnu / reproduction Philippe Duprat.

\section{La reprise de l'après-guerre : I'ancien bagne rasé (1945-1964)}

Après la guerre, Zodiac relance son activité avec la fabrication de bateaux pneumatiques, ainsi que la SNCASO, qui devient Sud-Aviation en 1957 : ces sociétés investissent les bâtiments encore exploitables de la partie sud de l'arsenal, notamment la zone des anciennes grandes forges. Une photographie aérienne du tout début des années 1950 montre la limite de l'implantation industrielle dans ce secteur: la rive droite de la Charente demeure à l'état d'abandon (bassin d'échouage totalement envasé, anciennes cales obliques de constructions réduites à l'état de vestiges à peine visibles) ainsi que les murs de l'ancien bagne ruiné, encore en élévation [fig. 14]. 
Figure 14



Vue aérienne de la partie sud de l'arsenal, vers 1950.

(c) André Bouclaud / reproduction archives municipales de Rochefort.

43 La destruction définitive de ces vestiges du bagne intervient dès 1952, avec l'installation de la base américaine de l'OTAN qui occupe massivement tout le secteur libre ou en déshérence. Les bâtiments viables sont investis et remis d'aplomb et les ruines sont rasées; c'est le sort des murs de l'ancien bagne qui disparaissent à cette occasion, ainsi que ceux de la salle Saint-Jacques et ceux qui subsistaient encore des magasins particuliers, bordant l'actuelle rue de l'Arsenal. Toute la surface est aplanie, rationalisée pour faire place nette, et quadrillée de voies tracées géométriquement. De nombreuses structures provisoires neuves surgissent [fig. 15]. L'ancien bassin d'échouage est même dévasé pour servir de base aux véhicules amphibies. 
Figure 15



Vue aérienne de la partie sud de l'arsenal, vers 1955 : base américaine

(c) André Bouclaud / reproduction archives municipales de Rochefort.

Dès lors, après le départ des Américains en 1964, la zone sud est prête pour la restructuration des lieux par l'industrie aéronautique ${ }^{33}$. La succession des périodes d'occupation, des destructions et des réaménagements a donc porté un coup fatal aux bâtiments de l'ancien bagne qui, dès 1952, disparaissent de la mémoire collective.

Ce n'est pas le cas de la zone nord, en partie ruinée, qui va bénéficier d'une reconquête patrimoniale autour d'un monument destiné à devenir l'emblème de Rochefort: la Corderie royale $^{34}$.

\section{La réindustrialisation massive du secteur sud et l'oubli définitif du bagne}

À partir de 1964, Zodiac et Sud-Aviation commencent à se partager les vastes espaces laissés par les Américains et encore largement inexploités en 1970. C'est la création de la « Z. I. de l'Arsenal » en 1971 qui donne une impulsion à cette extension. Sud-Aviation devenu SOCEA ${ }^{35}$ en 1970, SOCEA-SOGERMA ${ }^{36}$ en 1988 puis EADS-SOGERMA ${ }^{37}$ en 2000 ne cesse de développer ses activités (liées à l'émergence des avions Airbus) et d'accroître son implantation au bord de la Charente ${ }^{38}$.

Parallèlement, Zodiac, installé plus au sud, poursuit son développement et investit l'emprise de l'ancien bagne. Une partie des vestiges rasés est irrémédiablement détruite par le creusement d'un espace souterrain au nord.

EADS-SOGERMA acquiert en 2010 les surfaces occupées par Zodiac, qui a cessé son activité en 2009, et restructure son implantation dans cette zone. En 2015, EADSSOGERMA devient STELIA Aerospace, qui augmente encore sa surface en effectuant de nouveaux travaux de réaménagement sans contrôle archéologique ${ }^{39}$. Les modules industriels ${ }^{40}$ recouvrent désormais presque totalement l'emprise de l'ancien bagne [fig. 16]. 
Figure 16



Vue aérienne de la partie sud de l'arsenal (extrait), 2014.

DAO sur le Websig géohistorique sur Rochefort : https://geoapps.huma-num.fr/adws/app/

0101e6d0-628e-11 e8-8be3-dfOfcbe237ee/ [lien valide novembre 2021].

49 La voie qui desservait la partie sud de l'arsenal (de l'Avant-garde - extrême sud - à la porte du Soleil - donnant sur le centre-ville) coupait en deux l'implantation de la SOCEA-SOGERMA : rachetée par la Ville en 1979, la partie nord de la route a été cédée par la suite à l'entreprise. Le reste de la voie, nommée « rue de l'Arsenal » en 1989, sépare actuellement le Magasin général ${ }^{41}$ de l'ancien bagne [fig. 17] : son tracé en traverse la cour, et sa large bordure ouest recouvre les restes de l'ancienne fontaine des forçats et de quelques bâtiments dont les cachots et le contrôle administratif. 




Vue actuelle de la rue de l'Arsenal, 2019.

(C) A. Dalançon (Société de Géographie de Rochefort).

Ces vestiges enterrés demeurent facilement accessibles à des fouilles archéologiques qui pourraient, à l'avenir, être éventuellement prescrites. Aucune indication (panneau, plaque) n'existe actuellement pour rappeler l'existence de ce monument si bien que, hormis quelques spécialistes, personne à Rochefort n'est à ce jour capable de localiser l'ancien bagne ${ }^{42}$.

\section{Le bagne de Rochefort : mythe, légende noire et choix mémoriels}

51 C'est donc la conjonction d'une multiplicité de facteurs qui peut expliquer un tel oubli. Le temps, bien sûr, en premier lieu : 168 ans ont passé depuis la fermeture du bagne. Mais aussi l'éloignement du centre vital de l'arsenal et de ses monuments prestigieux, au premier rang desquels la Corderie. Troisième raison objective de l'effacement du bagne : sa reconversion immédiate en bâtiment industriel, tradition qui ne s'est jamais démentie jusqu'à ce jour, avec le poids croissant de l'industrie aéronautique depuis 1971. S'y ajoutent les deux destructions successivement opérées par les Allemands en 1944 puis par les Américains en 1952.

Il existe toutefois une cause moins immédiatement repérable qui a joué un rôle capital dans cet effacement. Elle est liée à une légende noire du bagne de Rochefort, phénomène qui trouve son origine dans la publication par l'ancien bagnard François Vidocq (1775-1857), en 1828, de ses Mémoires, dont l'énorme succès fut aussitôt relayé par l'ouvrage du journaliste Maurice Alhoy (1802-1856), Les Bagnes. Rochefort (1830), évoqué plus haut. Là-dessus se greffe la création par Balzac, dès 1834, dans Le Père Goriot, d'un personnage romanesque qui deviendra l'un des géants de la Comédie humaine: le bagnard protéiforme et diabolique Vautrin / Collin, alias «Trompe-laMort ». Le mythe est ainsi lancé ; quant au bagne de Rochefort (réservé à partir de 1828 aux forçats des longues peines - c'est-à-dire les plus endurcis), c'est à partir de cette 
époque qu'il accède chez Balzac ${ }^{43}$ à la dignité mythique d'établissement infernal, dont nul ne peut s'évader ni revenir vivant. Dans les représentations ${ }^{44}$, cette image de l'enfer, accentuée par la surmortalité liée aux fièvres des marais et aux pneumopathies, restera collée au bagne de Rochefort.

Localement, on s'empressera donc de l'oublier, d'enfouir cette honte originelle, d'effacer cette tache de l'histoire et du paysage par une reconversion industrielle immédiate. Depuis l'après-guerre, les municipalités qui se sont succédé jusqu'à ce jour n'ont cessé de mettre en avant la réindustrialisation d'une ville sinistrée depuis la fermeture de l'arsenal en 1927, argument repris régulièrement et accentué par le développement constant de l'aéronautique, secteur vital à Rochefort, et concentré dans la partie sud de l'arsenal.

Parallèlement, dans les années 1970, les choix mémoriels se sont portés sur les éléments prestigieux du patrimoine, ceux qui ont fait la "gloire » de la ville-arsenal (une gloire largement mythique elle aussi) : la reconquête a concerné prioritairement la partie nord, dominée par la Corderie royale, tandis qu'au sud n'ont été préservées que certaines structures qui avaient échappé aux destructions de 1944, comme le Magasin général.

L'ancien bagne - aux structures par ailleurs peu spectaculaires - a fait les frais de cette conjonction de facteurs particulièrement défavorables à toute conservation.

\section{NOTES}

1. Et très peu d'objets en dehors d'une écuelle de fontaine (musée Hèbre, Rochefort), de la cloche du bagne et de quelques objets réalisés par les forçats (musée national de la Marine, Rochefort), en particulier la pendule du forçat Dubois. DUHÉ Jean-Marc \& ROLAND Denis, « La pendule du forçat Dubois ", Roccafortis, n 61, 2018, p. 22-37.

2. Très nombreux plans de l'arsenal : Vincennes, SHD, série DD2 et fonds Nivard.

3. Archives manuscrites du bagne : matricules des forçats (série 0 ), documents administratifs et correspondances diverses (série E en particulier), services de la santé de la Marine, etc.

4. Archives photographiques en particulier.

5. Réalisé par l'université de La Rochelle et consultable sur Géolitto via geoapps.huma-num.fr [lien valide en octobre 2021].

6. L'archéologie professionnelle n'existe à Rochefort que depuis 2008, où l'évaluation archéologique des Fonderies (réalisée par Léopold Maurel) a déterminé la protection de toute la ville intra muros. Depuis les dernières destructions liées aux extensions de 2010 et 2015 (EADSSOGERMA et STELIA), l'ensemble du secteur de l'ancien arsenal, zone industrielle comprise, a été inscrit en zonage archéologique protégé. En quelques années, les diagnostics, suivis dans certains cas de fouilles, se sont multipliés et ont permis d'importantes découvertes, dont l'une, toute récente, d'une tour de l'ancien château médiéval de Rochefort - aujourd'hui totalement disparu.

7. 20 juin 1763 , lettre de Michel-Joseph Froger de l'Éguille (commandant de la Marine à Rochefort) au duc de Choiseul (ministre de la Marine), SHD Rochefort, I E 28, lettres du ministre, 1763. 
8. Abandon de la construction d'une frégate (1758) et de deux vaisseaux (1759) sur les trois commencés, ainsi que l'incendie accidentel, à la fin de 1758, d'un grand bâtiment des Magasins particuliers, qui ne sera jamais réédifié faute de moyens.

9. 6 mai 1766, lettre du duc de Praslin (ministre de la Marine) à Charles Claude de Ruis-Embito (intendant de la Marine à Rochefort), SHD Rochefort, I E 179, Dépêches de la Cour - Marine, 1766.

10. Même s'il convient de relativiser la fréquence de la cordelle (en moyenne 2 à 4 halages annuels).

11. JOANNIC-SETA Frédérique, Le Bagne de Brest. Naissance d'une institution carcérale au siècle des Lumières, Presses universitaires de Rennes, coll. « Histoire », 2000, chapitre V, p. 101-118.

12. À Brest, les forçats sont logés dans un bâtiment monumental dominant la rive droite de la Penfeld, édifié entre 1749 et 1751, image emblématique du "bagne des Lumières » (André Zysberg), et détruit par les bombardements de 1944.

13. «Taulas » ou « taulars » : immense plancher surélevé (voir fig. 2).

14. Convoi de condamnés enchaînés à destination du bagne.

15. Auxquels se joindront 11 condamnés supplémentaires en octobre, soit 539 hommes en tout.

16. Commissaire du bagne de Rochefort de 1808 à 1815 . Voir « Note du commissaire Millet sur la chiourme de Rochefort en 1813 », Roccafortis, n 61, 2018, p. 38-44, disponible en ligne http:// socgeo-rochefort.fr/documents/fichiers/905_attach.pdf [lien valide en octobre 2021]. Note remise à la Société de Littérature, Sciences et Arts par M. Millet, commissaire de Marine, original manuscrit (archives de la Société de géographie de Rochefort).

17. Décompte des constructions navales à Rochefort (ensemble de l'article) établi à partir de : PIERRE Joël, Les Constructions navales à Rochefort, Brouage, Soubise et Tonnay-Charente, Paris, Le Croît vif, 2003, p. 33-65.

18. Pendant la guerre d'Amérique, les effectifs des trois bagnes dépassent les 6000 condamnés.

19. Les chiffres subissent d'importantes fluctuations en fonction des besoins : ils redescendent brutalement à 402 en 1783, une fois la victoire acquise. Pour le délicat problème des effectifs de la chiourme (fortes variations dans le courant de chaque année), nous avons travaillé à partir des ouvrages suivants: "Note du commissaire Millet sur la chiourme de Rochefort en 1813 ", Roccafortis, $\mathrm{n}^{\circ}$ 61, 2018 ; GRATEAU Louis-Hippolyte, Esquisse médicale du bagne de Rochefort, thèse de médecine (faculté de médecine de Montpellier, 12 juillet 1833), Montpellier, Auguste Ricard Imprimerie, 1833, p.55, extrait disponible en ligne https://gallica.bnf.fr/ark:/12148/ bpt6k314050n?rk=21459;2 [lien valide en octobre 2021] ; OYAUX Pierrick, « Le Bagne de Rochefort (1766-1852)», mémoire de maitrise d'histoire, Aix-Marseille, 1981 (SHD Rochefort, RO T26) ; SOCIÉTÉ DE GÉOGRAPHIE DE ROCHEFORT, Le Bagne de Rochefort (1766-1852), catalogue d'exposition (20 septembre 2019-avril 2020, musée de la Vieille Paroisse, Rochefort), Société de géographie de Rochefort, 2019, p. 16-17.

20. 1100 hommes en 1799, 1200 en 1801, 1600 en 1803 ; puis les chiffres oscillent entre 1279 et 1612 jusqu'en 1814.

21. Selon l'expression d'A. Zysberg. Voir ZYSBERG André, « Des galères aux bagnes : continuité, rupture et évolution d'une institution pénale en France », in CENTRE D’ÉTUDES ROCHEFORTAISES MARITIMES, Rochefort et la mer. Les Bagnes des arsenaux, Rochefort, 2014, t. 31, p. 38.

22. Ainsi les 34 Miquelets catalans «forçats espagnols [...] provenant du fort de Figuières » ( Note du commissaire Millet sur la chiourme de Rochefort en 1813 », Roccafortis, $n^{\circ}$ 61, 2018, p. 43) et déportés au bagne de Rochefort en 1811. 22 survivants seront détachés de la chaîne en 1814.

23. La morbidité est importante au bagne de Rochefort, où les fièvres intermittentes et les maladies pulmonaires provoquent une forte surmortalité. 13272 forçats meurent au bagne entre 1766 et 1852 sur les 25950 qui y séjournèrent, soit plus de la moitié. D’où la mauvaise réputation de cet établissement et une inefficacité chronique liée à l'indisponibilité régulière d'une large partie de l'effectif immobilisée à l'hôpital ou dans les salles de convalescence et d'hébergement. 
24. SOCIÉTÉ DE GÉOGRAPHIE DE ROCHEFORT, Le Bagne de Rochefort (1766-1852), catalogue d'exposition (20 septembre 2019-avril 2020, musée de la Vieille Paroisse, Rochefort), Société de géographie de Rochefort, 2019, p. 20-21.

25. DUHÉ Jean-Marc, «Les murs du bagne de l'arsenal de Rochefort », in CENTRE D'ÉTUDES ROCHEFORTAISES MARITIMES, Rochefort et la mer. Les Bagnes des arsenaux, Rochefort, 2014, t. 31, p. 53-98.

26. Règlement du 30 avril 1818, promulgué par Charles Toussaint Pouyer, intendant du port de Rochefort de 1817 à 1824.

27. Deux forçats réunis par une chaîne, attachée autour du cou.

28. Forçats découplés, ne portant plus que la «chaussette » (anneau de cheville habituellement rattaché à la chaîne).

29. ALHOY Maurice, Les Bagnes. Rochefort, Paris, Gagniard, 1830, disponible en ligne https:// gallica.bnf.fr/ark:/12148/bpt6k57505973.texteImage [lien valide en octobre 2021].

30. Les Tablettes des deux Charentes, $\mathrm{n}^{\circ} 14,18$ février 1852, disponible en ligne https:// www.retronews.fr/journal/les-tablettes-des-deux-charentes/18-fevrier-1852/645/2708509/2

[lien valide en octobre 2021].

31. Société nationale des constructions aéronautiques du Sud-Ouest.

32. Sous l'Occupation, la zone interdite est une bande de terre côtière de 20 à $30 \mathrm{~km}$ de large, qui s'étend de Dunkerque à Hendaye. Cette zone fait l'objet d'une règlementation spéciale en octobre 1941.

33. L'extrémité sud sera affectée à l'École des fourriers de la Marine dès 1964 (jusqu'en 2002).

34. Les travaux de réhabilitation, commencés en 1976 et achevés en 1987, s'élargissent dans la foulée à l'ensemble du secteur : hôtel de la Corderie royale (ancienne direction de l'Artillerie), Bassins I et II, remparts nord, jardin des Retours, etc.

35. Société charentaise d'équipements aéronautiques.

36. Société girondine d'entretien et de réparations de matériel.

37. European Aeronautic Defence and Space Company. Le site de Rochefort acquiert une dimension européenne à partir du Xxi ${ }^{\mathrm{e}}$ siècle.

38. En créant quatre gros ensembles jointifs d'ateliers entre 1972 et 2003.

39. Voir note 5.

40. Actuellement ceux de l'entreprise SATYS SELINGS \& PAINTING FRANCE, spécialisée dans les peintures pour l'aéronautique.

41. Les Magasins généraux (et certains éléments des Magasins particuliers) ont été réhabilités entre 1988 et 1998.

42. Une exposition a été organisée par la Société de géographie de Rochefort au Musée archéologique de la Vieille Paroisse, de septembre 2019 à fin mars 2020, accompagnée d'une brochure de 64 pages : SOCIÉTÉ DE GÉOGRAPHIE DE ROCHEFORT, Le Bagne de Rochefort (1766-1852), catalogue d'exposition, Société de géographie de Rochefort, 2019.

43. BALZAC Honoré de, Illusions perdues (1837-1843) et Splendeurs et misères des courtisanes (1838-1847).

44. La légende noire est prolongée et amplifiée par les mémoires de bagnards qui se multiplient à la suite de Vidocq (Anthelme Collet, Jean-Joseph Clémens...), et par le succès de certains textes d'inspiration populaire (les feuilletons des journaux, l'Histoire des bagnes depuis leur création jusqu'à nos jours de Pierre Zaccone, publié en 1876. Voir la réédition de 1878 via https://gallica.bnf.fr/ ark:/12148/bpt6k6115200z.texteImage [lien valide en octobre 2021]. 


\section{RÉSUMÉS}

Le bagne de Rochefort, à la fois territoire clos et dispositif éclaté dans l'arsenal et la ville, est actuellement un patrimoine disparu: la mémoire des lieux - voire celle de l'institution - a sombré dans l'oubli le plus total, à la différence notable du monument le plus emblématique de la ville, la Corderie royale. Pourtant les deux bâtiments étaient dans le même état de délabrement après les destructions allemandes de 1944.

L'objet de l'article est de retracer l'histoire de ce lieu pénitentiaire qui a accueilli 25000 forçats entre 1766 et 1852, dont plus de la moitié sont morts en détention. Les bâtiments initiaux, conçus dans l'urgence pour recevoir 600 hommes, furent rapidement doublés durant la guerre d'Indépendance américaine, puis aménagés et complétés, à mesure de l'augmentation des effectifs, jusqu'à l'extension maximale, sous la Restauration.

Le développement de la vapeur et du fer, ainsi que le manque de qualification de la main-d'œuvre des forçats, conduisent à la fermeture du bagne de Rochefort, le premier à être supprimé en France en 1852.

Les locaux du bagne sont alors reconvertis en bâtiments industriels dans un arsenal qui retrouve un second souffle. Cette vocation industrielle, après l'intermède de l'Occupation et celui de la base américaine de l'OTAN, ne se dément pas de nos jours : ce sont des bâtiments du secteur aéronautique (Satys) qui couvrent actuellement le site historique d'un bagne dont Balzac a contribué à faire un lieu mythique.

The penal prison of Rochefort was a confined space with different sites spread in the arsenal and in town. It is now a lost and forgotten heritage. The memory of the site itself and of the institution has sunk into complete oblivion, unlike the most emblematic monument of Rochefort, the Corderie Royale (rope manufacture) which was in the same state of extreme dilapidation after being blown up in 1944.

This article aims at focusing on the history of Rochefort harbour prison which had up to 25,000 inmates over a period of 86 years (1766-1852). More than 50\% died in detention.

The original buildings which had been hastily put up could not receive more than 600 men. During the American War of Independence more buildings had to be put up to increase the prison capacity. They were gradually extended and fitted out as the number of inmates grew until the prison maximum capacity was reached.

In the $19^{\text {th }}$ century the rapid growth of steam engines and trains and a lack of qualified labour among the detainees led the prison to close down in 1852. The old prison was converted into industrial premises giving the arsenal a new start. The industrial vocation of the place still continues today after the interludes of wartime German occupation and the post war US NATO basis. Air industry buildings now stand on the historical site of the prison transformed into a myth by the French novelist Balzac.

INDEX

Mots-clés : bagne, Rochefort, arsenal, reconversion industrielle, mémoire, mythe

Keywords : penal colony, Rochefort, arsenal, industrial redevelopment, memory, myth 


\section{AUTEUR}

\section{PHILIPPE DUPRAT}

Agrégé de Lettres classiques

Président de la Société de Géographie de Rochefort

philduprat@wanadoo.fr 